\title{
Tetrabromobisphenol A adsorption by active carbon: influential factors and kinetics
}

Xiaoli $\mathrm{Su}^{1}$, Mingming Wang ${ }^{1}$, Songwen Yang ${ }^{1}$, Feiyun Sun*1, Jiaju Dong ${ }^{2}$,Yiping Rong ${ }^{2}$, Bin Zhang ${ }^{2}$, Desong Hong ${ }^{2}$

${ }^{1}$ Harbin Institute of Technology, Shenzhen, 518055, China

${ }^{2}$ Shenzhen New Land Tool Planning \& Architectural Design Co.Ltd, Shenzhen 518172, China

\begin{abstract}
Frequency detection and environmental risk of Tetrabromobisphenol A ( TBBPA) in aqueous system attracted many concerns. Nevertheless, mutual interactions between TBBPA and natural or synthetic chemical materials has not be clearly revealed. In this study, influential factors, adsorption behavior and mutual dynamism of active carbon (AC) and TBBPA were comprehensively explored. It was found that chemical sorption efficiency of AC reached more than $90 \%$. The adsorption behavior can be depicted by Langmuir and Freundlich isotherms, whose kinetic could be well described by pseudo-first-order kinetic model. The chemisorption of AC is mainly single molecular layer mode for TBBPA adsorption, during which presence of $\mathrm{SO}_{4}{ }^{2-}, \mathrm{HCO}_{3}{ }^{-}$and humic acid significantly inhibited TBBPA sorption capability. Proper temperature and $\mathrm{pH}$ promoted the adsorption efficiency of TBBPA. The obtained results demonstrated that $\mathrm{AC}$ adsorption is one feasible and effective technique for enhanced treatment technique for TBBPA containing wastewater.
\end{abstract}

Keyword. Tetrabromobisphenol A (TBBPA); Active carbon; Adsorption

\section{Introduction}

Recently, considerable attention has been paid to Tetrabromobisphenol A (TBBPA, $\mathrm{C}_{15} \mathrm{H}_{12} \mathrm{Br}_{4} \mathrm{O}_{2}$, CAS Registration No.79-94-7), as its occurrence and transportation in water environment. TBBPA is one typical brominated flame retardants (BFRs) that has been widely used in the manufacture of printed circuit board (PCB), due to its advantages, eg. less additive dosage, good flame retardancy and thermal stability[1]. However, TBBPA has the properties of bioaccumulation and toxicity, result to potential threat to human and animal health [2]. Thus, an effective treatment method for TBBPA removal in water is highly expected. Common techniques for TBBPA removal include photocatalytic degradation,

\footnotetext{
*Corresponding author: sun_fy@ hit.edu.cn
} 
adsorption, biodegradation and chemical oxidation, ect. [3-8]. However, after the wastewater containing TBBPA was treated by the wastewater treatment system, there are still many residues with rather low concentration (generally less than $10 \mathrm{ng} \mathrm{L}^{-1}$ ), which could not be effectively removed by traditional biological method and advanced oxidation process.

Activated carbon (AC) is a commonly used adsorbent in adsorption technology, because of its large number of micropores and specific surface area, and there with high physical adsorption capacity. Application of AC in wastewater treatment has been characterized as high treatment efficiency and stable effluent quality, especially for the organic pollutants that are difficult to be removed by biological and chemical methods[9]. In this work, adsorption behavior of TBBPA by AC was explored and the effect of initial concentration, dosage of $\mathrm{AC}$, system temperature, $\mathrm{pH}$ value, coexisting substances were also examined. The results may insight that adsorption of TBBPA by AC is a feasible and effective technique and give technical support for the removal of typical BFRs in the existing wastewater treatment facilities and their upgrading.

\section{Materials and Methods}

\subsection{Adsorbent}

The basic parameters of the AC coconut shell used in the experiment were shown in Table 1. The $\mathrm{AC}$ was firstly washed thoroughly with distilled water, and then soaked in deionized water for $24 \mathrm{~h}$ and dried in the oven for adsorption tests.

Table1. The parameter of AC

\begin{tabular}{ccccccc}
\hline Parameter & $\begin{array}{c}\text { Particle size } \\
(\mathrm{mesh})\end{array}$ & $\begin{array}{c}\text { Density } \\
\left(\mathrm{g} \mathrm{cm}^{-3}\right)\end{array}$ & $\begin{array}{c}\text { Intensity } \\
(\%)\end{array}$ & $\begin{array}{c}\text { Iodineadsorption } \\
\left(\mathrm{mg} \mathrm{g}^{-1}\right)\end{array}$ & $\begin{array}{c}\text { Ash content } \\
(\%)\end{array}$ & $\mathrm{pH}$ \\
\hline $\begin{array}{c}\text { numerical } \\
\text { value }\end{array}$ & $10 \sim 28$ & $0.45 \sim 0.54$ & 97 & 1050 & 3 & $5 \sim 9$ \\
\hline
\end{tabular}

\subsection{Adsorption experiments}

The batch adsorption experiments were carried out in erlenmeyer flask $(250 \mathrm{~mL})$ that contained suitable quantity of AC, and $100 \mathrm{~mL}$ TBBPA stock solutions under varied environmental condition. Series of flasks were sealed and placed in a thermostatic oscillator for stable adsorption. Varied TBBPA concentration, dosage of $\mathrm{AC}$, initial $\mathrm{pH}$ value, temperature and the concentration of co-existing substance were kept for different adsorption tests. In kinetics experiments, $2 \mathrm{~mL}$ solution was sampled regularly 0 , $0.5,1,2,4,8,12,24 \mathrm{~h}$, respectively, until the adsorption equilibrium reached. After centrifugal $15 \mathrm{~min}$ with $8000 \mathrm{r} \mathrm{min}^{-1}$, the concentration of TBBPA in the supernatant was analyzed by Ultra Performance Liquid Chromatography, UPLC (Waters H-Class, USA). The adsorption amounts and removal efficiency could be calculated by Eq. 1 and 2 .

$$
\begin{aligned}
Q_{t} & =\frac{\left(C_{0}-C_{t}\right) V}{1000 W} \\
\mathrm{P} & =\frac{C_{0}-C_{t}}{C_{O}} \times 100 \%
\end{aligned}
$$

where $\mathrm{Q}_{\mathrm{t}}\left(\mathrm{mg} \mathrm{g}^{-1}\right)$ is the adsorption amount at time $\mathrm{t} ; \mathrm{C}_{0}$ and $\mathrm{C}_{\mathrm{t}}$ in $\mathrm{mg} \mathrm{L}^{-1}$ are the initial concentration and concentration at time $\mathrm{t}\left(\mathrm{mg} \mathrm{L}^{-1}\right) ; \mathrm{P}(\%)$ is the removal rate of TBBPA at time $\mathrm{t} ; \mathrm{V}(\mathrm{mL})$ is the solution volume while $\mathrm{W}(\mathrm{g})$ is the total summation of sorbent. 


\subsection{Optimization of TBBPA adsorption by AC}

The initial concentration of TBBPA, AC dosage and AC mean size, initial $\mathrm{pH}$ value, temperature, and the concentration of coexisting substance, were conducted to optimized the TBBPA adsorption condition parameters. The reaction temperature was $25 \pm 1{ }^{\circ} \mathrm{C}$, the initial concentration of TBBPA was 1 $\mathrm{mg} \mathrm{L}^{-1}$, the dosage of AC was 0.1, 0.2, 0.5, 1.0 and $1.5 \mathrm{~g}$, respectively. The removal effect of TBBPA with different amount of active carbon was examined.

\subsection{Kinetics and isotherm experiments}

In this study, quasi-primary kinetic model, quasi-secondary kinetic model and Elovich kinetic model were used to fit the kinetic data of adsorption of TBBPA, so as to determine the most suitable kinetic model. Analysis of adsorption kinetics is helpful to shed light on the adsorption mechanism. The pseudofirst order kinetics model was expressed as Eq. 3.

$$
\log \left(Q_{e}-Q_{t}\right)=\log Q_{e}-\left(\frac{K_{1}}{2.303}\right) \times t
$$

Where $Q_{e}\left(\mathrm{mg} \mathrm{g}^{-1}\right)$ represents adsorption at equilibrium; $Q t\left(\mathrm{mg} \mathrm{g}^{-1}\right)$ represents amount of adsorbent at time $\mathrm{t}, \mathrm{t}(\mathrm{min})$ and $k_{I}\left(1 \mathrm{~min}^{-1}\right)$ is the rate constant.

The pseudo-second order kinetics model is defined as Eq. 4.

$$
\frac{t}{Q_{t}}=\frac{1}{\left(k_{2} Q_{e}^{2}\right)}+\frac{t}{Q_{e}}
$$

where $k_{2}\left(\mathrm{~g} \mathrm{mg}^{-1} \mathrm{~min}^{-1}\right)$ is the pseudo-second order rate constant

The Elovich model is defined as Eq. 5.

$$
Q_{t}=\frac{1}{\beta} \ln (\alpha \beta)+\frac{1}{\beta} \ln (t)
$$

Where $\alpha\left(\mathrm{mg} \mathrm{g}^{-1} \mathrm{~min}^{-1}\right)$ is the initial adsorption rate and $\beta\left(\mathrm{mg} \mathrm{g}^{-1} \mathrm{~min}^{-1}\right)$ is the desorption constant, which is the role of AC surface functionality in the adsorption of TBBPA.

Adsorption isotherms were used to describe the distribution of adsorbent molecules between solid and liquid phases. Isotherm studies were performed at designated initial TBBPA concentrations (10、15、 20、25、30、32 and $35 \mathrm{mg} \mathrm{L}^{-1}$, respectively) at $25 \pm 1^{\circ} \mathrm{C}$ temperatures. The dosage of $\mathrm{AC}$ was taking out in $1 \mathrm{~g}$. The adsorption isotherm of TBBPA in AC was fitted by Langmuir and Freundlich models, according to Eq. 6 and 7, respectively.

$$
\frac{1}{Q e}=\frac{1}{Q e}+\frac{1}{k_{L} Q_{\max }} \cdot \frac{1}{c_{e}}
$$

Where $Q_{e}\left(\mathrm{mg} \mathrm{g}^{-1}\right)$ is the adsorption amount of Langmuir adsorption equilibrium; $C_{e}\left(\mathrm{mg} \mathrm{L}^{-1}\right)$ is the representative of initial concentration of TBBPA; $k_{L}\left(\mathrm{~L} \mathrm{mg}^{-1}\right)$ is the Langmuir adsorption equilibrium constant which related to adsorption capacity, and $Q_{\max }$ is the maximum adsorption capability.

$$
\log \left(Q_{e}\right)=\frac{1}{n} \lg \left(C_{e}\right)+\lg \left(K_{L}\right)
$$

Where $\mathrm{n}$ and $K_{F}$ are the empirical constants which represent the relative adsorption capacity of adsorption strength and bond energy respectively. A straight line is obtained by drawing $\lg Q_{e}$ to $\lg C_{e}$ as shown in Figure 3 (c), so as to calculate the maximum adsorption volume $\mathrm{n}$ and the adsorption constant $K_{L}$. 


\section{Results and discussion}

\subsection{Influential factors}

Impact of temperature on the adsorption of TBBPA by AC was investigated. As shown in Fig. 1 (a), the removal effeciency of TBBPA under the four temperature as $10,20,30$ and $40{ }^{\circ} \mathrm{C}$ were measured. With the increase of temperature, the higher the adsorption efficiency and the faster the removal rate of TBBPA on the AC are, indicating that the adsorption of TBBPA on the AC is more favorable at mediumhigh temperature. It is speculated that the adsorption process of TBBPA on AC needs heat energy at different temperatures, so the proper temperature increase is conducive to the reaction.
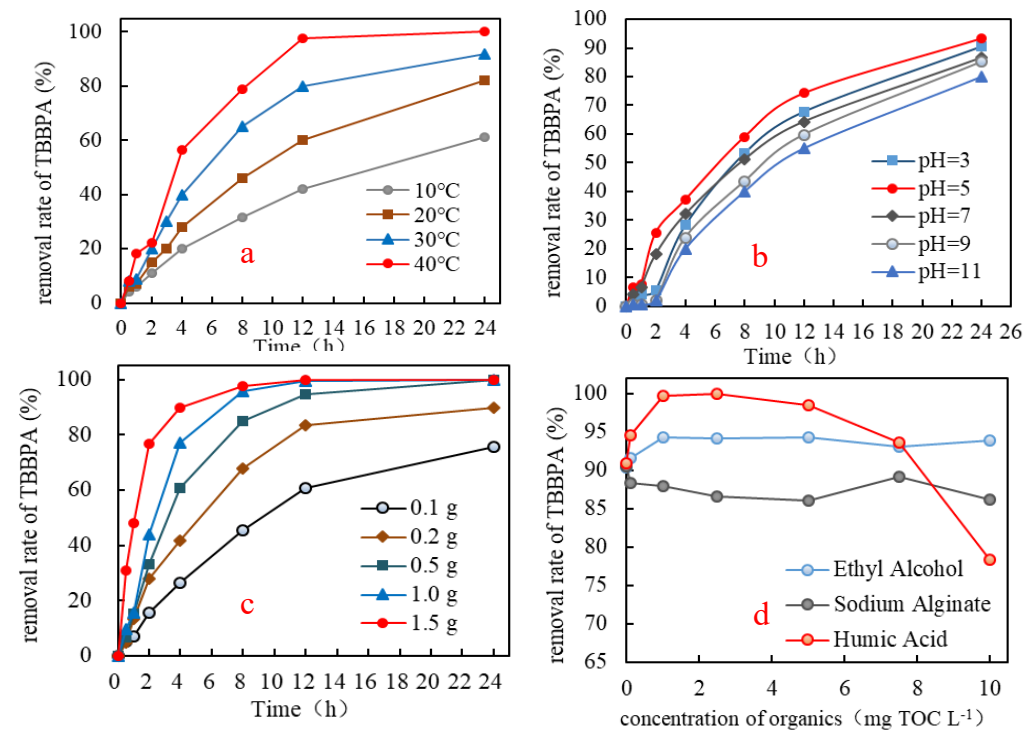

Fig. 1. The removal effect of different environment factor AC on TBBPA changes with time. (a)Temperature;

(b) $\mathrm{pH}$ value; (c) Dosage of AC; (d) Organic dosage

Changing the $\mathrm{pH}$ value of the solution may lead to the change of the morphology of TBBPA and the chemical properties of the surface of the AC, thus affecting the adsorption. In the experiment, sodium hydroxide solution and hydrochloric acid are used to adjust the $\mathrm{pH}$ value of the solution, the $\mathrm{pH}$ value conditions of multiple groups of solutions are set to study the influence on the adsorption of TBBPA by the AC. The reaction is carried out under the condition of $25 \pm 1^{\circ} \mathrm{C}$ with an initial concentration of TBBPA of $1 \mathrm{mg} \mathrm{L}^{-1}$ and dosage of the AC of $200 \mathrm{mg}$, The test results are shown in Fig.1 (b), Acidic conditions were favorable for the adsorption and removal of TBBPA by $\mathrm{AC}$. When $\mathrm{pH}=5.0$, the removal rate of TBBPA by AC reached 93\%.Alkaline conditions inhibited the removal of TBBPA, and the removal rate was the lowest when $\mathrm{pH}=11.0$, only $80 \%$. The reason may be related to the existing form of TBBPA, because the $\mathrm{pKa} 1$ and $\mathrm{pKa} 2$ of TBBPA are 7.5 and 8.5 , respectively. When the $\mathrm{pH}$ value of the solution is less than 7, TBBPA mainly exists in the molecular state, so the active carbon has a very good adsorption and removal effect on it. When the $\mathrm{pH}$ value of the solution is greater than $\mathrm{pKa}$, the ionization of TBBPA mainly exists in the form of anion, which increases the degree of ionization and dissolution in water and reduces the hydrophobicity of TBBPA, thus reducing the interaction between TBBPA and active carbon. The adsorption capacity decreased with the increase of $\mathrm{pH}$ value. In addition, the electrostatic repulsion between the negative charge $\left(\mathrm{pH}<\mathrm{pH}_{\mathrm{pcz}}=8.82\right)$ on the surface of $\mathrm{AC}$ and the anion of TBBPA can also 
reduce the adsorption capacity of AC on TBBPA.

Under the condition of initial TBBPA concentration of $1 \mathrm{mg} \mathrm{L}^{-1}$, the effect of the amount of adsorbent on AC adsorption of TBBPA was investigated. As demonstrated in Fig.1(c), the removal rate increased while reaction time was opposite to removal effeciency as the rise up of AC dosage. The adsorption efficiency of $0.5 \mathrm{~g}$ system is $94.6 \%$ for $12 \mathrm{~h}$ and that of $1.5 \mathrm{~g}$ system is no significant improvement but reduce reaction time in $4 \mathrm{~h}$ as the dosage changed from 0.5 to $1.5 \mathrm{mg} \mathrm{L}^{-1}$. The increase in the amount of adsorption is mainly due to increased adsorption point and enlarged surface area due to increase dosage of AC [10]. That make magnify the opportunity of AC come into contact with TBBPA, the reaction will be accelerated and AC quickly and massively absorb TBBPA in solution. The removal rate is relatively stable with the carbon dosage further increased. This may be Limited concentration of AC result in overlapping effective adsorption points [11]. Therefore, in practical application, we should choose the appropriate dosage of AC according to the actual situation.

The co-existing organics in wastewater may affect the adsorption process of TBBPA by AC. Based on the investigation of wastewater quality, the adsorption capacity of TBBPA by AC under the influence of three coexistent organic compounds (ethanol, sodium alginate and humic acid) for 24 hours was compared and the influence of the dosage of co-existent substances on adsorption capacity by AC was explored. As shown in Fig. 1 (d), ethanol has almost no effect in the removal of TBBPA and the removal rate is kept at about $92 \%$. The reason is that ethanol is easily soluble in water, which does not block the pore diameter of the $\mathrm{AC}$ and does not affect the adsorption rate of TBBPA on the AC. Sodium alginate can inhibit the removal of TBBPA. after adding sodium alginate, the removal rate of sodium alginate by $\mathrm{AC}$ is reduced by $5 \%$, which may be due to the strong hydrophilicity of sodium alginate, which dissolves in water to form a thick and uniform solution, adheres to the surface of $\mathrm{AC}$, occupies a part of adsorption sites, resulting in pore blockage, thus reducing the adsorption performance of AC on TBBPA. The removal rate of TBBPA in aqueous solution will be promoted by the appropriate amount of humic acid

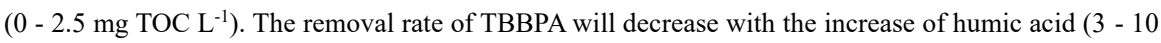

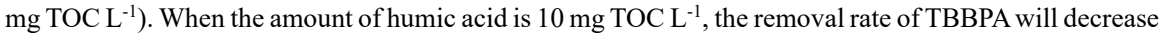
by $13 \%$. Humic acid is a kind of macromolecular substance, and its molecular weight $\left(10^{2}-10^{4} \mathrm{dal}\right)$ is large, which makes it difficult to enter into the pore diameter of AC. The adsorption capacity of AC is limited, and the adsorption of TBBPA by AC is not affected when the concentration is small. Increasing the concentration of humic acid will inevitably cause some micropores to be blocked, which will reduce the adsorption effect of AC on TBBPA. At the same time, the oxygen-containing functional groups on the surface of humic acid may produce repulsive force on the adsorption of TBBPA[12].

\subsection{The effect of inorganic ions}

The common cations $\left(\mathrm{NH}_{4}{ }^{+} 、 \mathrm{Cu}^{2+}\right)$ and anions $\left(\mathrm{SO}_{4}{ }^{2-}, \mathrm{HCO}_{3}{ }^{-}\right)$in wastewater may affect the adsorption of TBBPA on AC. This part of the experiment is based on the investigation of the actual wastewater quality. Under the same conditions, the initial concentration of TBBPA is $1 \mathrm{mg} \mathrm{L}^{-1}$, the dosage of $\mathrm{AC}$ is $200 \mathrm{mg}$, and the reaction temperature is $25 \pm 1^{\circ} \mathrm{C}$. It can be seen from Fig. 2 (a) and (b) that the presence or absence of $\mathrm{NH}_{4}{ }^{+}$and $\mathrm{Cu}^{2+}$ in the adsorption system and the concentration change have little effect on the adsorption of TBBPA by AC. Cations do not compete with TBBPA in adsorption on AC. When the dosage of $\mathrm{SO}_{4}{ }^{2-}$ exceeds $0.5 \mathrm{mg} \mathrm{L}^{-1}$, it can promote the removal of TBBPA in Figure 2 (c). When $\mathrm{SO}_{4}{ }^{2-}$ was not added, the removal rate of TBBPA was $95 \%$; when $\mathrm{SO}_{4}{ }^{2-}$ was added at $1.5 \mathrm{mg} \mathrm{L}^{-1}$, the removal rate of TBBPA was increased by $4 \%$. It can be seen from Fig.2(d) that $\mathrm{HCO}_{3}{ }^{-}$has a significant effect on the removal effect of TBBPA. When the dosage of $\mathrm{HCO}_{3}{ }^{-}$increased from 0 to $1.5 \mathrm{mg} \mathrm{L}^{-1}$, 
removal effeciency about TBBPA decreased from $97 \%$ to $91 \%$. While the dosage of $\mathrm{HCO}_{3}{ }^{-}$was $3 \mathrm{mg} \mathrm{L}$ ${ }^{1}$, the removal rate reached $97 \%$, which was the same as when $\mathrm{HCO}_{3}{ }^{-}$was not added. The co-existing $\mathrm{HCO}_{3}{ }^{-}$can weaken the electrostatic attraction in the solution system, and may also compete with TBBPA for the active sites of the activated carbon, resulting in the decrease of adsorption capacity[13]. As the ionic strength increases with the increase of $\mathrm{HCO}_{3}{ }^{-}$concentration, the increase of its interaction leads to the decrease of $\mathrm{HCO}_{3}{ }^{-}$effective concentration with the decrease of activity coefficient and the decrease of the competitive ability with the active carbon of TBBPA[14].
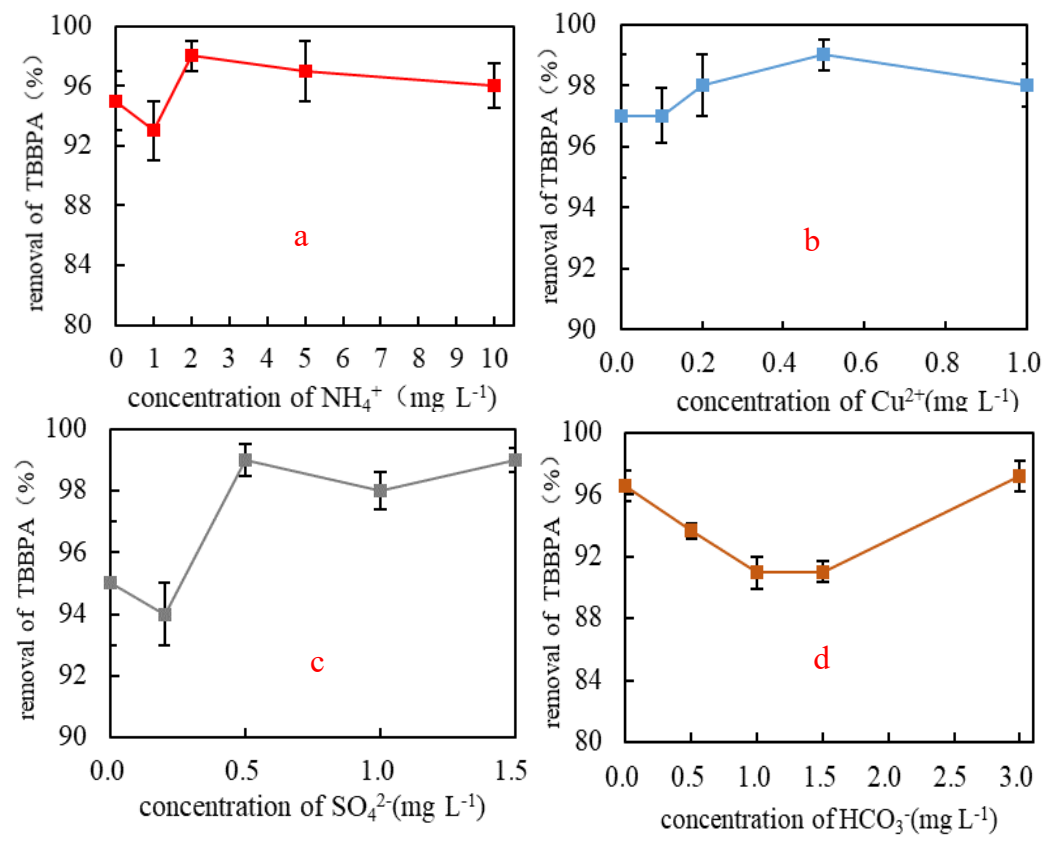

Fig. 2. Effect of inorganic ions on adsorption of TBBPA by AC, where (a) means the effect of $\mathrm{NH}_{4}^{+}$; (b) means the effect of $\mathrm{Cu}^{2+}$; (c) means the effect of $\mathrm{SO}_{4}{ }^{2-}$;(d) means the effect of $\mathrm{HCO}_{3}{ }^{-}$.

\subsection{Adsorption kinetics}

The adsorption kinetic parameters can be calculated from the adsorption kinetic data, which can explain the adsorption mechanism of adsorbents in the liquid phase to some extent[15].The adsorption kinetics was analyzed using pseudo-first-order(Fig.3(a)), pseudo-second-order (Fig.3(b)) and Elovich models(Fig.3(c)). The rate limiting step of pseudo first order kinetics is determined by the diffusion of particles, while the rate limiting step of pseudo second order kinetics is determined by the number of active sites. 

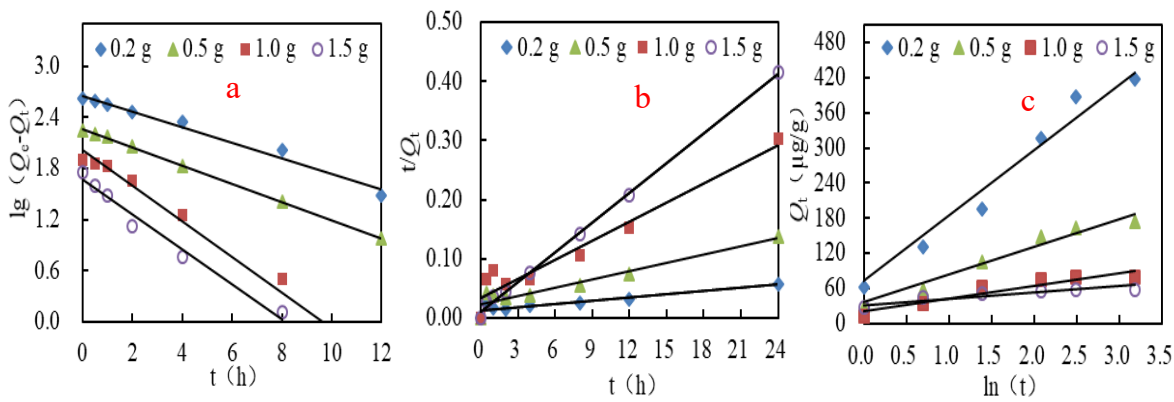

Fig. 3. Quasi-primary, quasi-secondary and Elovich kinetic models of TBBPA adsorption by activated carbon. (a) represent Quasi-primary kinetic models; (b) Quasi- secondary kinetic models ; (c) Elovich kinetic models

Table 2 lists the kinetic parameters of TBBPA adsorption on AC calculated by equation (3), (4) and (5) . The linear correlation coefficient $R^{2}$ of quasi-first-order kinetic equation is larger than that of quasisecond-order and Elovich order, so that compared with the pseudo-first order model, this model the model can reflect the adsorption mechanism of TBBPA on AC more comprehensively. In addition, it can be seen from Table 2 that with the increase of the dosage of $\mathrm{AC}$, the adsorption rate constant tends to increase, that is to say, the larger the dosage of $\mathrm{AC}$ is, the faster the adsorption rate of TBBPA reaches equilibrium. This also indicates that chemisorption plays an important role in activated carbon, which is due to the effective electron transfer and adsorption between the active sites on the surface of TBBPA and $\mathrm{AC}$.

Table 2. Kinetic parameters of TBBPA adsorption on AC

\begin{tabular}{cccccccc}
\hline \multirow{2}{*}{$\begin{array}{c}\text { Adsorbent } \\
\text { Quantity }\end{array}$} & \multicolumn{2}{c}{ Pseudo-first-order } & \multicolumn{2}{c}{ Pseudo-second-order } & \multicolumn{3}{c}{ Elovich model } \\
\cline { 2 - 7 } 0.2 & $K_{1}$ & $R^{2}$ & $K_{2}$ & $R^{2}$ & $R^{2}$ & $\alpha$ & $\beta$ \\
\cline { 2 - 7 } 0.5 & 0.0035 & 0.9822 & 0.0050 & 0.8609 & 0.9739 & 0.0036 & 0.1483 \\
1 & 0.0041 & 0.9993 & 0.0167 & 0.9114 & 0.9717 & 0.0017 & 0.3517 \\
1.5 & 0.0080 & 0.9864 & 0.0567 & 0.9366 & 0.9316 & 0.0010 & 0.7650 \\
\hline
\end{tabular}

\subsection{Adsorption isotherm of TBBPA adsorbed by AC}

With the rise up of equilibrium concentration, the adsorption capacity gradually increases and gradually approaches equilibrium (Fig.4 (a)), indicating that the adsorption site of AC is covered and TBBPA is more and more difficult to reach the surface adsorption site. To further explore the mechanism of adsorption, The adsorption isotherm of TBBPA in AC was fitted by Langmuir and Freundlich models(Fig.4 (b)\&(c)). Adsorption isotherms can be used to describe the distribution of adsorbent molecules between solid and liquid phases. The isotherm model parameters were listed in Table 3. the adsorption of TBBPA by AC, the linear correlation coefficient $\left(R^{2}\right)$ obtained by Langmuir model fitting is slightly higher than that of Freundlich model(Table 3). This shows that the energy distribution of the active site on the surface of the AC is uniform. In other words, TBBPA molecules are mainly adsorbed on the external surface of the $\mathrm{AC}$ in the form of a single molecular layer[16]. Once the adsorption site on the surface of $\mathrm{AC}$ is occupied, no further adsorption can occur at this location, which is consistent with the results in Table 3. It can also be concluded from table 4 that the maximum adsorption capacity of AC for TBBPA is $39.37 \mathrm{mg} \mathrm{g}^{-1}$, and $\mathrm{N}=1.1$, so AC is easy to adsorb TBBPA in solution. 

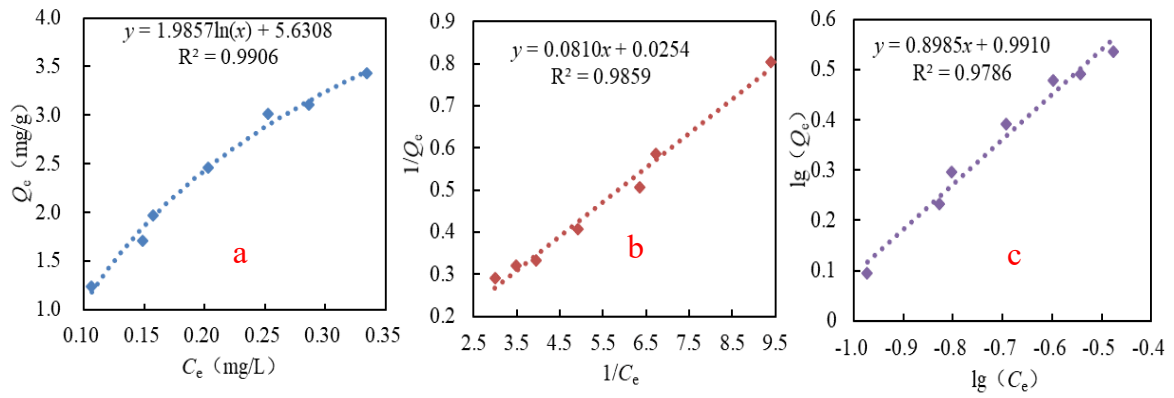

Fig. 4. Adsorption characteristics of activated carbon. (a) Isothermal adsorption curve of TBBPA by AC; (b)Langmuir isothermal adsorption model; (c)Freundlich isothermal adsorption model.

Table 3. Langmuir and lreundlich model parameters of TBBPA adsorption on AC

\begin{tabular}{ccccccc}
\hline \multicolumn{3}{c}{ Langmuir } & & \multicolumn{3}{c}{ Freundlich } \\
\cline { 1 - 2 } \cline { 5 - 7 }$R^{2}$ & $Q_{\max }\left(\mathrm{mg} \mathrm{g}^{-1}\right)$ & $K_{\mathrm{L}}\left(\mathrm{L} \mathrm{mg}^{-1}\right)$ & & $R^{2}$ & $K_{\mathrm{F}}\left(\mathrm{mg}^{1-1 / \mathrm{n}} \mathrm{L}^{1 / \mathrm{n}} \mathrm{g}^{-1}\right)$ & $n$ \\
\hline 0.9859 & 39.37 & 0.3136 & & 0.9786 & 9.79 & 1.10 \\
\hline
\end{tabular}

\section{Conclusions}

The removal rate of TBBPA by AC can reach more than $90 \%$. The pseudo-first-order kinetic equation and Langmuir model can better describe the adsorption behavior of coconut shell activated carbon to TBBPA. The adsorption of AC to TBBPA is mainly monolayer adsorption, and the maximum adsorption capacity is $39.37 \mathrm{mg} \mathrm{g}^{-1}$. The presence of $\mathrm{SO}_{4}{ }^{2-}, \mathrm{HCO}_{3}{ }^{-}$and humic acid significantly inhibited the removal of TBBPA by AC. Appropriate temperature and $\mathrm{pH}$ could promote the adsorption effect of TBBPA.Therefore, the removal of TBBPA from aqueous phase by coconut shell activated carbon is an effective and feasible technology.

\section{Acknowledgements}

This work was financially supported by the Shenzhen Science and Technology Funding Project [grant number JSGG20170414101900541 and JCYJ20170816102318538]; the National Natural Science Foundation of China [grant numbers 51678183, 51408149] and the Project supported by Guangdong Natural Science Foundation [grant number 2017A030313285].

\section{References}

[1] J.w. Zhu,S. Diao, C.B Liu. Brominated flame retardants [J]. Science and technology information, 25,26-26(2012).

[2] Y.C. Jin, L.H. Li, X.L. Luo, et al. Research progress on degradation and toxicity of TBBPA [J]. Journal of minzu university of China, 2,5-10(2010).

[3] Y.Wu, Y.Wang, T.Pan, et al. Oxidation of TBBPA (TBBPA) by peroxymonosulfate: The role of insitu formed $\mathrm{HOBr}[\mathrm{J}]$. Water Research, 169,115-202(2020).

[4] Y. Li, X. Yang, X. Li. The progress on degradation technology of TBBPA[J]. Chemical industry and environmental protection, 34(04), 326-331(2014).

[5] J. Shen, G. Huang, C. An, et al. Removal of TBBPA by adsorption on pinecone-derived ACs: Synchrotron FTIR, kinetics and surface functionality analyses[J]. Bioresource Technology, 247, 812- 
$820(2018)$.

[6] Q. Han, W.Y. Dong, H. Wang, et al. Degradation of TBBPA by ozonation: Performance, products, mechanism and toxicity[J]. Chemosphere, 235, 701-712(2019).

[7] Y.Y. Cui, H.B. Ren, C.X. Yang, et al. Facile synthesis of hydroxyl enriched microporous organic networks for enhanced adsorption and removal of TBBPA from aqueous solution[J]. Chemical Engineering Journal, 373, 606-615(2019).

[8] Q. Zhou, Y. Wang, J. Xiao, et al. Preparation of magnetic core-shell $\mathrm{Fe}_{3} \mathrm{O}_{4} @$ polyaniline composite material and its application in adsorption and removal of TBBPA and decabromodiphenyl ether[J]. Ecotoxicology and Environmental Safety, 183, 109471(2019).

[9] P. Peng, Y.H. Lang, X.M. Wang. Adsorption behavior and mechanism of pentachlorophenol on reed biochars: $\mathrm{pH}$ effect, pyrolysis temperature, hydrochloric acid treatment and isotherms[J]. Ecological Engineering, 90, 225-233(2016).

[10] V. K. Garg, R. Gupta, A. BalaYadav, et al. Dye removal from aqueous solution by adsorption on treated sawdust[J]. Bioresource Technology, 89(2), 121-124(2003).

[11] C. Namasivayam, D. Prabha, M. Kumutha. Removal of direct red and acid brilliant blue by adsorption on to banana pith[J]. Bioresource Technology, 64(1): 77-79(1998).

[12] K. Wang. Study on the adsorption of GAC and the removal of HAAs in water by AOP $\mathrm{s}_{\mathrm{s}}$ and mechanism[D]. Chongqing University(2005).

[13] X. Zheng, Y. Zhou, X. Liu, et al. Enhanced adsorption capacity of MgO/N-doped active carbon derived from sugarcane bagasse[J]. Bioresource Technology, 297: 122413(2020).

[14] G. Zelmanov, R. Semiat. The influence of competitive inorganic ions on phosphate removal from water by adsorption on iron $\left(\mathrm{Fe}^{3+}\right)$ oxide/hydroxide nanoparticles-based agglomerates[J]. Journal of Water Process Engineering, 5: 143-152(2015).

[15] N. Aoki, M. Nishikawa, K. Hattori. Synthesis of chitosan derivatives bearing cyclodextrin and adsorption of p-nonylphenol and bisphenol A[J]. Carbohydrate Polymers, 52(3): 219-223(2003).

[16] Y. Zhu. Overview and development suggestion of chemicals for printed circuit board[D]. Tianjin chemical industry, 2:21-24(2002). 